\title{
HETEROSIS, HABILIDAD COMBINATORIA Y DIVERSIDAD GENÉTICA EN HÍBRIDOS COMERCIALES DE MAÍZ (Zea mays L.)1
}

\author{
Alfredo de la Rosa ${ }^{2}$, Humberto de León ${ }^{2}$, Gaspar Martínez², Froylan Rincón ${ }^{2}$
}

RESUMEN

Heterosis, habilidad combinatoria y diversidad genética en híbridos comerciales de maíz (Zea mays L). El conocimiento de la habilidad combinatoria, diversidad genética $\mathrm{y}$ heterosis del germoplasma de un programa de mejoramiento es esencial para desarrollar híbridos y variedades. Con base en lo anterior se realizó un estudio con el siguiente objetivo. 1. Identificar híbridos con valor genético para iniciar un programa de mejoramiento para producir híbridos y variedades. 2. Demostrar que es factible crear un programa de mejoramiento a partir de híbridos de maíz. Las estimaciones de habilidad combinatoria fueron obtenidas por el método IV de Griffing (1956) y las distancias genéticas por el método de Troyer (1988) Las cruzas más sobresalientes fueron AS910 X AS4450 y PP9538 X AS948 con un rendimiento de 17.538 y $17.463 \mathrm{t} / \mathrm{ha}$ respectivamente, estas mismas obtuvieron los valores más altos de ACE. Las cruzas con las heterosis más altas en base a la media de los padres fueron PP9539 X AN453 y PP9603 X PP9539 con 11,35 y 11,13\% respectivamente. Los híbridos con los valores más altos ACG fueron PP9539 y AN447 con 1.168 y 0.684 t/ha respectivamente, y los mejores en heterosis PP9539 y A7500 con 2.269 y 1.170 respectivamente. Los híbridos más relacionados fueron AN450 y AS910 con una distancia genética de 0,033, y una heterosis de $-47,68 \%$; en tanto que los menos relacionados fueron AN454 y A7500 con una distancia de 1.418 y una heterosis de $12,26 \%$.

\section{ABSTRACT}

Heterosis, GCA, SCA and gentic diversity of 13 hybrids of maize (Zea mays L.). Knowledge about the combining ability, genetic diversity and heterosis of germoplasm from a maize breeding program is essential for hybrids and varieties development. The objetives of this study were (1) To identify commercial hybrids with high genetic value (GCA and heterosis) useful for initiating a maize breeding program to produce and improved hybrids, varieties and synthetics; (2) To demonstrate that is feasible to create a maize breeding program from commercial hybrids. Estimates of general (GCA) and specific combining ability (SCA) were obtained using the method IV of Griffing (1956) and the genetic distances were estimated according to the method suggested by Troyer (1988). The best crosses were AS910 X AS4450 and PP9538 X AS948 having a yield of 17.538 and $17.463 \mathrm{t} / \mathrm{ha}$ respectively; the same crosses had the highest values of SCA. The crosses with the highest values of heterosis over the midparent were PP9539 X AN453 and PP9603 X PP9539 with 11,35 and 11,13 percent respectively. The highest positive values of GCA effects were obtained from hybrids PP9539 and AN447 with 1.168 and 0.684 t/ha respectively;. and the hybrids with the best heterosis were PP9539 ( 2.269 ) and A7500 ( 1.170 ). The most related hybrids were AS910 and AN450 with a genetic distance of 0,033 and heterosis of $-47,68$ percent whereas the most diverse were AN454 and A7500 with a genetic distance of 1.418 and heterosis of $12,26 \%$.

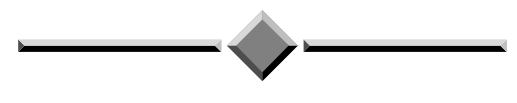

\section{INTRODUCCIÓN}

El tener conocimiento de la diversidad genética, heterosis y habilidad combinatoria general y especifica del germoplasma de un programa de mejoramiento es esencial para diversos objetivos como: 1) desarrollar híbridos y o variedades, 2) Crear variabilidad genética 3) Evitar vulnerabilidad genética, 4) Implementar pro- gramas de selección recurrente. Una forma de estimar estos parámetros genéticos es por medio de los diseños dialélicos como los propuestos por Griffing (1956) o por el diseño de Gardner y Eberharth (1966), por otro lado existen varias formas de medir la diversidad genética como el propuesto por Garza et al (1962) y el propuesto por Troyer (1988) los cuales funcionan en base al resultado de cruzas, autofecundaciones y heterosis de

1 Trabajo presentado en la XLV Reunión Anual del PCCMCA, Guatemala. 1999.

2 Departamento de Fitomejoramiento. Universidad Autónoma Agraria Antonio Narro. Saltillo Coahuila, México Tel/Fax (84) 1773-61. 
los materiales utilizados, otra forma es por medio de marcadores genéticos moleculares como RFLPs, RAPDs, AFLPs etc. que han sido ampliamente utilizados para medir la diversidad genética y agrupamiento de líneas (Lee et al., 1989; Smith et al., 1990; Melchinger 1990 y Munn 1994) así como de híbridos (Smith et al., 1989) una ventaja de este último método es que se pueden analizar una gran cantidad de individuos, sin embargo se ha llegado a la conclusión de que la distancia medida por marcadores es de uso limitado para predecir patrones heteroticos entre líneas de maíz. En este tipo de estudios siempre se han utilizado materiales como líneas, poblaciones y variedades sintéticas, en este estudio se utilizaron híbridos comerciales partiendo de las siguientes suposiciones: si los híbridos están formados por líneas que han pasado por un largo proceso de selección, como los es el sobrevivir a alrededor de 4 a 5 autofecundaciones, y en cada una de estas etapas ser seleccionadas hacia varias características como. rendimiento, resistencia a enfermedades e insectos, acame de raíz y de planta, por lo tanto cuando llegan a la etapa final han dejado atrás bastante carga genética, así que los híbridos que llegan a liberarse en forma comercial portan bastantes genes deseables y pocos genes indeseables, por están razones, los híbridos pueden ser usados exitosa y ventajosamente en programas de mejoramiento. Los objetivos del presente trabajo fueron los siguientes. 1. Identificar híbridos comerciales con valor genético para iniciar un programa de mejoramiento para producir híbridos y variedades. 2. Demostrar que es factible crear un programa de mejoramiento a corto plazo a partir de híbridos.

\section{MATERIALES Y MÉTODOS}

El trabajo de se llevó a cabo en el Instituto Mexicano del Maíz "Dr. Mario E. Castro Gil” (I.M.M) de la Universidad Autónoma Agraria Antonio Narro, en el ciclo 96-97.

El material genético utilizado en este trabajo de investigación (Cuadro 1), fue el resultado de un dialélico entre 13 híbridos comerciales de maíz ( 78 cruzas) más los 13 híbridos progenitores lo cual da un total de 91 genotipos.

La siembra se efectuó para cada una de las tres localidades usadas de manera independiente de acuerdo a las fechas de siembra establecidas regionalmente. En Celaya, Gto. $\left(\mathrm{L}_{1}\right)$ se realizó el 30 de abril de 1996; Celaya, Gto $\left(\mathrm{L}_{2}\right) 30$ de junio, y Sandia el Grande N.L. $\left(\mathrm{L}_{3}\right)$ el 1 de mayo del mismo año. La parcela experimental en las tres localidades fue de dos surcos de 4,41 $\mathrm{m}$ de largo por $0,75 \mathrm{~m}$ de ancho dando una área de
Cuadro 1. Material Genético utilizado en el presente trabajo.Coahuila, México. 1996

\begin{tabular}{rllc}
\hline & HÍBRIDO & COMPAÑIA & No. de Líneas \\
\hline 1 & C220 & CARGILL & 3 \\
2 & C221 & CARGILL & 3 \\
3 & A7500 & ASGROW & 3 \\
4 & PP9603 & DEKALB & 2 \\
5 & PP9539 & DEKALB & 2 \\
6 & PP9538 & DEKALB & 2 \\
7 & AN447 & I.M.M & 3 \\
8 & AN454 & I.M.M & 4 \\
9 & AN450 & I.M.M & 4 \\
10 & AN453 & I.M.M & 4 \\
11 & AS910 & ASPROS & 4 \\
12 & AS4450 & ASPROS & 3 \\
13 & AS948 & ASPROS & 3 \\
\hline
\end{tabular}

parcela útil de $6.615 \mathrm{~m}^{2}$, con 21 plantas por surco y dos repeticiones por tratamiento. La siembra del material experimental se llevó al cabo en forma manual, depositando dos semillas por golpe, para posteriormente aclarear a una planta por mata y así asegurar al número optimo de plantas. El desarrollo del cultivo fue bajo condiciones de riego, con la aplicación oportuna de plaguicidas cuando fue necesario.

Celaya, Gto.- Se sitúa a 20032' latitud Norte; 1004' longitud Oeste, con una altitud de 1754 msnm; con una temperatura media anual de $20,6^{\circ} \mathrm{C}$ y una precipitación pluvial anual de $597.3 \mathrm{~mm}$.

Sandia el Grande, N. L.- Se sitúa a 24049' latitud Norte; $100^{\circ} 04^{\prime}$ longitud Oeste, con una altitud de 1654 msnm; con una temperatura media anual de $18,6^{\circ} \mathrm{C}$ y una precipitación pluvial anual de 365,0 mm.

La fórmula de fertilización aplicada (N-P-K), fue 180-90-00, la cual se distribuyo en dos partes; la primera al momento de la siembra (90-90-00), y la segunda en el primer cultivo. El número de riegos aplicados fue variable, esto estuvo en función de los requerimientos específicos de cada localidad.

\section{Análisis dialélico}

El valor genético de los híbridos comerciales actualmente en uso, para ser usados como fuente de germoplasma en mejoramiento se evaluó a través del comportamiento de las cruzas y de los progenitores mismos realizando un análisis de aptitud combinatoria general y especifica, basándose en el análisis propuesto por Griffing (1956), utilizando el método IV (el cual solo incluye las cruzas directas $F_{1}$ ). El modelo lineal aditivo fue el siguiente: 


$$
Y i j=m+g_{i}+g_{j}+s_{i j}+e_{i j}
$$

Donde:

$Y_{i j}=$ Valor fenotipico observado de la cruza con progenitores i y $\mathrm{j}$

$=$ Media general.

$\mathrm{g}_{\mathrm{i}}, \mathrm{g}_{\mathrm{j}}=$ Efecto de ACG del progenitor $\mathrm{i}, \mathrm{j}$

$\mathrm{s}_{\mathrm{ij}}=$ Efecto de ACE de la cruza $\left({ }_{\mathrm{ij}}\right)$

$\mathrm{e}_{\mathrm{ij}}=$ Error experimental.

Así como también mediante el análisis general de efectos genético (método II) de Gardner y Eberhart (1966) En este análisis el modelo matemáticos de los híbridos o poblaciones $\left(\mathrm{Y}_{\mathrm{j}} \mathrm{o} \mathrm{Y}_{\mathrm{j}}\right.$ ) y las cruzas $\left(\mathrm{Y}_{\mathrm{j} j}\right.$ ، $)$ se expresa de la manera siguiente:

$$
\begin{aligned}
& Y_{j}=\mu_{v}+v_{j} \\
& Y_{j} ،=\mu_{v}+v_{j} ، \\
& Y_{j j} ،=\mu_{v}+{ }_{-}\left(v_{j}+v_{j} \cdot\right)+h_{j j} ،
\end{aligned}
$$

Donde:

$\mu_{\mathrm{v}}=$ Media de los padres.

$\mathrm{v}_{\mathrm{j}} \mathrm{y}_{\mathrm{j}}$ ، $=$ Efecto de los híbridos ${ }_{\mathrm{j}} \mathrm{y}_{\mathrm{j}}$ 。

$\mathrm{h}_{\mathrm{j} j}$ ، = Efecto de la heterosis correspondiente a la $\operatorname{cruza}_{\mathrm{j}} \mathrm{y}_{\mathrm{j}}$ 。

El efecto de heterosis fue subdividido de la manera siguiente:

$$
h_{j j} \cdot=h+h_{j}+h_{j} \cdot+s_{j j} \text { }
$$

Donde:

$\mathrm{h}=$ heterosis promedio.

$\mathrm{h}_{\mathrm{j}}=$ heterosis varietal contribuida por la variedad $_{\mathrm{j}}$

$\mathrm{h}_{\mathrm{j}^{\mathrm{c}}}=$ heterosis varietal contribuida por la variedad $\mathrm{j}^{\text {‘ }}$

$\mathrm{s}_{\mathrm{j} j}$; = efecto de la heterosis correspondiente a la cru

$\mathrm{za}_{\mathrm{j}} \mathrm{y}_{\mathrm{j}}$ 。

La estimación de la diversidad genética entre híbridos se estimó por medio de la formula de Troyer (1988) de la siguiente forma:

$$
G D=1-\frac{H-C}{H-S}
$$

Donde:

GD = Diversidad genética entre híbridos .

$\mathrm{H}=$ Comportamiento promedio de los híbridos.

$\mathrm{C}=$ Comportamiento de la cruza híbrido por híbrido.

$\mathrm{S}=$ Comportamiento de la progenie de los híbridos autofecundados.
La estimación de la heterosis entre los híbridos se hizo en base al progenitor medio de la siguiente forma.

$$
H=\frac{F_{1}-M P}{M P}
$$

Donde:

$\mathrm{H}=$ Heterosis

$\mathrm{F}_{1}=$ La media de la primera generación de la cruza entre los dos progenitores.

$\mathrm{MP}=$ Media de los progenitores.

El método de análisis de agrupamiento (Cluster Analysis) que se usó para el agrupamiento de los híbridos en base a diversidad genética y heterosis fue el UPGMA Esta estrategia computa la distancia promedio para formar un agrupamiento $\left({ }_{i j}\right)$. Este proceso usa las distancias de todos los pares de individuos en el cluster $\left(\mathrm{n}_{\mathrm{i}}, \mathrm{n}_{\mathrm{j}}\right)$ las distancias entre el grupo $\left(_{\mathrm{ij}}\right)$ y otro cluster $\mathrm{H}$ es obtenido por:

$$
D(i j) H=\frac{\sum_{i} \sum_{j} D i k}{N(K) N(H)}
$$

Donde Dik es la distancia entre individuos en el cluster $\left({ }_{i j}\right)$ e individuos en el cluster $\mathrm{H}$. NK y $\mathrm{NH}$ es el numero de datos en el cluster $\left(_{\text {i j }}\right.$ ) y $\mathrm{H}$, respectivamente. los parámetros usados por la formula combinatoria son: $\mathrm{a}_{\mathrm{i}}=\mathrm{a}_{\mathrm{j}}=0.5 ; \mathrm{b}=0 ; \mathrm{y} \mathrm{g}=-0,5$ (Everitt, 1980; Lance y Williams 1969).

\section{RESULTADOS Y DISCUSIÓN}

En base a la hipótesis planteada en el presente trabajo, de que existe diferente fondo genético entre los híbridos utilizados, se discute los siguiente.

En el Cuadro 2 se presentan los cuadrados medios de los análisis de varianza dialélicos combinados para las cuatro características agronómicas evaluadas durante el ciclo primavera verano en el año de 1996, en la localidad de Celaya, Gto. en dos fechas de siembra y en la localidad de Sandia, N.L. Se puede observar que hubo diferencias significativas $(\mathrm{p} \leq 0,01)$ entre localidades para todas las características evaluadas, lo que indica que las condiciones climáticas y edáficas para cada localidad son diferentes; también se observaron diferencias significativas $(p \leq 0,01)$ entre cruzas para todas la características evaluadas, las cuales pueden ser atribuidas a la gran diversidad genética que presentan los progenitores, haciendo posible la identificación de cruzas 
Cuadro 2. Cuadrados medios de los análisis de varianza combinados a través de tres ambientes, dos ambientes en Celaya, Gto. y uno en Sandia, N.L. Coahuila, México. 1996-97.

\begin{tabular}{|c|c|c|c|c|c|c|}
\hline FV & GL. & $\begin{array}{l}\text { RENDIMIENTO } \\
\text { t/ ha }\end{array}$ & $\begin{array}{l}\text { ALTURA DE } \\
\text { PLANTA cm }\end{array}$ & $\begin{array}{c}\text { HUM. DE } \\
\text { GRANO \% }\end{array}$ & G.L. & $\underset{\text { d }}{\text { DIAS A FLOR }}$ \\
\hline LOC. & 2 & $2082,02 * *$ & $448715,118 * *$ & $13232,67 * *$ & 1 & $5971,875 * *$ \\
\hline REP/LOC. & 3 & 24,970 & 501,335 & 3,703 & 2 & 73,542 \\
\hline CRUZAS & 77 & $9,813 * *$ & $499,695 * *$ & $16,081 * *$ & 77 & $21,819 * *$ \\
\hline $\mathrm{ACG}$ & 12 & $25,425 * *$ & $959,328 * *$ & $37,286 * *$ & 12 & $115,085^{* *}$ \\
\hline $\mathrm{ACE}$ & 65 & $6,931 * *$ & $414,840 * *$ & $12,164 * *$ & 65 & $4,600 *$ \\
\hline CRUZAS X LOCS. & 154 & $8,653 * *$ & 324,54 & $10,569 * *$ & 77 & $4,784 * *$ \\
\hline ACG X LOCS. & 24 & $29,873 * *$ & $512,047 * *$ & $20,367 * *$ & 12 & $6,020 * *$ \\
\hline ACE X LOCS & 130 & $4,743 * *$ & 289,466 & $8,761 * *$ & 65 & 1,721 \\
\hline ERROR & 231 & 2,587 & 329,041 & 2,571 & 154 & 3,035 \\
\hline C.V. $(\%)$ & & 11,30 & 7,94 & 8,24 & & 2,16 \\
\hline D.M.S. $(5 \%)$ & & 1,820 & 20,52 & 1,814 & & 2,41 \\
\hline MEDIA. & & 14,235 & 228,38 & 19,4 & & 81,00 \\
\hline MÁXIMO & & 17,583 & 249,00 & 23,65 & & 87,00 \\
\hline MÍNIMO & & 11,945 & 207,00 & 13,4 & & 75,00 \\
\hline
\end{tabular}

*** Significativo al 0,05 y 0,01 niveles de probabilidad respectivamente.

tardías, precoces, de porte alto o bajo y sobre todo cruzas rendidoras.

Al desglosar la fuente de variación cruzas en ACG y ACE, se encontraron diferencias significativas $(\mathrm{p} \leq 0,01)$ entre efectos de ACG para todas las características evaluadas; diferencias significativas $(\mathrm{p} \leq 0,01)$ para ACE en las características rendimiento y humedad de grano y diferencias significativas $(\mathrm{p} \leq 0,05)$ para días a flor masculina.

La contribución a la varianza del rendimiento atribuible a las cruzas esta constituida por un 40,4 por ciento de efectos aditivos (ACG) y un 59,6 por ciento de efectos no aditivos (ACE). Esta superioridad de los efectos no aditivos sobre los aditivos puede ser debido a la heterosis resultante de las combinaciones híbridas entre los progenitores. Para las características altura de planta y humedad de grano se observaron mayores efectos del tipo no aditivo que aditivo, 70 por ciento de ACE y 30 por ciento de ACG para altura de planta y 64 por ciento ACE y 36 por ciento de ACG para humedad de grano. Estos resultados se deben a que en estos caracteres también existió el beneficio de la heterosis, como lo hubo en rendimiento; en lo que respecta a días a floración se observaron mayores efectos del tipo aditivo ACG (82 por ciento) que el no aditivo ACE (18 por ciento) a causa de que los híbridos utilizados tienen días a floración similar y no es mucho el beneficio de la heterosis. En este Cuadro 1 también se presentan el coeficiente de variación, la media, y los valores máximo y mínimo de las características evaluadas, donde se observa que los coeficientes de variación son aceptables para las características evaluadas y que existe una buena amplitud entre estas para seleccionar buenos genotipos.
Se encontraron también diferencias significativas $(\mathrm{p} \leq 0.01)$ para cruzas por localidad para todas las características evaluadas, indicando que las cruzas no tienen la suficiente información genética para amortiguar las condiciones de los ambientes donde fueron evaluadas, es decir, no tienen estabilidad y varía su posición de localidad en localidad, de tal modo que si en una localidad una cruza tuvo un buen resultado, en otra localidad tuvo un mal resultado, lo cual es importante para seleccionar cruzas que se adapten a cada ambiente o que se comporten bien en todos los ambientes donde fueron evaluadas, según el interés del mejorador.

Para la interacción ACG por localidad existieron diferencias significativas $(\mathrm{p} \leq 0,01)$ para todas las características evaluadas, en cambio para la interacción ACE por localidad solo existieron diferencias significativas $(\mathrm{p} \leq 0,01)$ para rendimiento y humedad de grano. La interacción ACG x Loc. revela la importancia de los efectos aditivos en las características mencionadas, indicando también que los efectos de ACG de los padres para estas características cambia de localidad en localidad y por lo tanto se deben seleccionar aquellos que tengan mejores efectos de ACG a través de los ambientes para las características evaluadas, en cuanto a ACE X Loc, las significancias para rendimiento y humedad de grano indican que las cruzas no mantienen su ACE a través de los ambientes; sin embargo, aunque existieron estas diferencias significativas, se observó que para rendimiento los efectos aditivos y no aditivos contribuyeron casi en forma equilibrada a las cruzas siendo estos 53,8 por ciento aditivos y 46,2 por ciento no aditivos; para altura de planta 25 por ciento aditivos, 75 por ciento no aditivos; para humedad de grano 30 por ciento aditivos y 
70 por ciento no aditivos y para días a flor 39 por ciento aditivos y 61 por ciento no aditivos.

Al particionar la suma de cuadrados en ACG y ACE, casi todos los análisis mostraron que la mayoría de la variación es atribuida a la ACE (efectos no aditivos), estos valores indican la importancia de los efectos de dominancia en las cruzas en la expresión del rendimiento, lo que concuerda con lo obtenido por Castro, (1964); Cortez, (1985) y González, (1996) quienes observaron que los efectos de dominancia contribuían más a la varianza de las cruzas con respecto al rendimiento.
En el Cuadro 3 se presentan los valores combinados del rendimiento, heterosis del rendimiento, ACE para las características de altura de planta días a floración y humedad de grano a la cosecha de la mejores 25 cruzas evaluadas en dos localidades de Celaya, Gto. y una localidad en Sandia, N.L., donde se observa que la media del rendimiento de los padres (15.605 $\mathrm{t} / \mathrm{ha}$ ) fue superior a la media de las cruzas (14.235 t/ha). El comportamiento de los híbridos en las tres localidades evaluadas indican que los híbridos de 3 y 4 líneas rinden mas que los híbridos de seis, siete y ocho líneas, también se observa que el mejor padre fue el híbrido AS910, con un rendimiento de

Cuadro 3. Valor combinado de rendimiento, heterosis del rendimiento y ACE para, rendimiento, altura de planta, días a flor y humedad de grano de las 25 mejores cruzas con base en rendimiento, incluyendo los padres evaluados Celaya, Gto. en dos fechas y en Sandia, N.L. en al año de 1996. Coahuila, México. 1996-97.

\begin{tabular}{|c|c|c|c|c|c|c|c|}
\hline \multicolumn{2}{|c|}{ Progenitores } & \multirow{2}{*}{$\begin{array}{c}\text { Rendimiento } \\
\text { t/ ha }\end{array}$} & \multirow{2}{*}{$\begin{array}{c}\text { ACE.Rend. } \\
\text { t/ ha }\end{array}$} & \multirow{2}{*}{$\begin{array}{c}\text { ACE.Altura } \\
\text { cm }\end{array}$} & \multirow{2}{*}{$\begin{array}{c}\text { ACE } \\
\text { DAF. d. }\end{array}$} & \multirow{2}{*}{$\begin{array}{l}\text { ACE } \\
\text { HG. } \%\end{array}$} & \multirow{2}{*}{$\begin{array}{c}\text { Heterosis } \\
\%\end{array}$} \\
\hline १ & $\sigma^{\prime}$ & & & & & & \\
\hline AS910 & AS4450 & 17,538 & 2,669 & $-1,681$ & $-1,577$ & $-0,402$ & 0,93 \\
\hline PP9538 & AS948 & 17,463 & 2,633 & 8,227 & $-0,645$ & $-1,174$ & 6,75 \\
\hline PP9539 & AN453 & 17,355 & 2,038 & 6,091 & $-0,582$ & 0,644 & 11,35 \\
\hline PP9539 & AN450 & 17,188 & 1,741 & 5,227 & 0,977 & $-2,174$ & 7,41 \\
\hline PP9539 & AN447 & 17,072 & 0,985 & $-17,363$ & 0,755 & 1,098 & 9,97 \\
\hline AN454 & AS948 & 16,052 & 1,445 & 2,091 & 0,159 & $-1,402$ & $-3,38$ \\
\hline AN447 & AS98 & 16,033 & 0,661 & 8,727 & 4,569 & $-0,811$ & $-8,81$ \\
\hline PP9539 & AS948 & 16,000 & 0,144 & 2,682 & $-0,864$ & $-0,129$ & 3,27 \\
\hline PP9539 & AN454 & 15,996 & 0,673 & 2,000 & $-0,655$ & 0,508 & 9,23 \\
\hline PP9603 & PP9539 & 15,952 & 1,225 & $-2,136$ & $-0,172$ & $-0,447$ & 11,13 \\
\hline AN450 & AS4450 & 15,929 & 1,546 & 2,318 & $-3,018$ & 1,189 & $-5,69$ \\
\hline PP9539 & AS910 & 15,624 & $-0,310$ & 11,727 & 0,168 & $-0,765$ & $-5,31$ \\
\hline $\mathrm{C} 220$ & AN447 & 15,618 & 1,315 & $-8,591$ & 0,536 & $-0,856$ & 5,33 \\
\hline PP9538 & AS910 & 15,594 & 0,687 & 4,772 & 0,537 & 0,189 & $-10,69$ \\
\hline PP9538 & AN447 & 15,420 & 0,359 & $-4,319$ & $-0,727$ & 0,053 & $-4,91$ \\
\hline AN447 & AS4450 & 15,314 & 0,291 & 7,227 & $-4,141$ & 0,462 & $-6,39$ \\
\hline AN454 & AS4450 & 15,243 & 0,985 & 12,091 & $-0,100$ & 0,871 & $-1,30$ \\
\hline PP9538 & AN453 & 15,059 & 0,767 & 10,636 & $-2,310$ & 0,598 & $-7,52$ \\
\hline $\mathrm{C} 220$ & AN453 & 14,994 & 1,461 & $-8,136$ & 1,050 & 0,189 & 1,29 \\
\hline A7500 & AS910 & 14,910 & 0,847 & 10,091 & 0,750 & $-1,947$ & $-5,61$ \\
\hline AN453 & AS4450 & 14,863 & 0,611 & $-3,318$ & 1,823 & 0,508 & $-9,71$ \\
\hline $\mathrm{C} 221$ & AS910 & 14,856 & 1,054 & $-1,727$ & $-0,155$ & $-0,583$ & $-13,48$ \\
\hline PP9538 & AN454 & 14,769 & 0,473 & $-6,955$ & 1,114 & 0,462 & $-3,77$ \\
\hline AN447 & AS910 & 14,758 & $-0,690$ & 10,772 & 0,700 & 0,553 & $-24,83$ \\
\hline A7500 & AN447 & 14,605 & 0,387 & $-11,000$ & $-1,364$ & 0,417 & $-0,40$ \\
\hline \multirow[t]{14}{*}{ MEDIA DE } & CRUZAS & 14,235 & & & & & \\
\hline & $\mathrm{C} 220$ & 12,233 & & & & & \\
\hline & $\mathrm{C} 221$ & 14,212 & & & & & \\
\hline & A7500 & 11,987 & & & & & \\
\hline & PP9603 & 14,949 & & & & & \\
\hline & РP9539 & 13,402 & & & & & \\
\hline & PP9538 & 15,016 & & & & & \\
\hline & AN447 & 17,339 & & & & & \\
\hline & AN454 & 15,636 & & & & & \\
\hline & AN450 & 18,426 & & & & & \\
\hline & AN453 & 17,367 & & & & & \\
\hline & AS910 & 19,505 & & & & & \\
\hline & AS4450 & 15,245 & & & & & \\
\hline & AS948 & 17,553 & & & & & \\
\hline MEDIA DE & PADRES & 15,605 & & & & & \\
\hline
\end{tabular}


$19.505 \mathrm{t} / \mathrm{ha}$, seguido por el AN450 con $18.426 \mathrm{t} / \mathrm{ha} \mathrm{y}$ el AS948 con 17.553 t/ha.

Las mejores cruzas para rendimiento fueron AS910 $\mathrm{X}$ AS4450 con un rendimiento de $17.538 \mathrm{t} / \mathrm{ha}$ que, a pesar de que los híbridos progenitores son de la misma compañía, tienen diferente información genética por lo cual produce el buen rendimiento observado; el segundo y el tercer lugar fue obtenido por las cruzas PP9538 X AS948 con 17.463 t/ha y PP9539 X AN453 con $17.365 \mathrm{t} / \mathrm{ha}$ también con un buen rendimiento, que es de esperarse ya que los dos híbridos involucrados en la cruza son de diferente compañía, y por lo tanto, existe diversidad genética entre estos híbridos.

Las cruzas que obtuvieron los tres valores más altos de ACE para rendimiento, fueron las mismas que obtuvieron los mejores rendimientos, estas son AS910 X AS4450, PP9538 X AS948 y PP9539 X AN453 con $2.669,2.633$ y $2.038 \mathrm{t} / \mathrm{ha}$ respectivamente; con respecto a altura de planta las mejores fueron PP9539 X AN 447 con $-17,363 \mathrm{~cm}$. y AN7500 X AN447 con $-11,00$ $\mathrm{cm}$; para días a floración las mejores cruzas fueron AN447 x AS4450 con -4,141 días y AN450 X AS4450 con -3,018 días, y para humedad de grano las mejores fueron PP9539 X AN450 con -2,174 por ciento y A7500 X AS910 con $-1,947$ por ciento. Las mejores cinco cruzas por su rendimiento, estuvieron formadas tres de ellas con tres híbridos de la UAAAN y dos híbridos de Dekalb y las restantes con tres híbridos de Aspros y uno de Dekalb.

Con respecto a la heterosis, las mejores valores fueron obtenidos por las cruzas PP9539 X AN453 con 11,35 por ciento además de una buena heterosis esta fue una de las mejores cruzas en cuanto a rendimiento, la segunda mejor cruza en cuanto a heterosis fue PP9603
X PP9539 con un valor de 11,13 por ciento en el sus híbridos progenitores a pesar de que pertenecen a la misma compañía con su comportamiento en cruza muestran que existe diversidad genética entre ellos, no siendo así para la cruza AN447 X AS910 la cual tuvo un valor de heterosis de $-24,83$ por ciento, demostrando que estos híbridos están relacionados genéticamente. Es importante señalar que de los mejores cinco híbridos con base en la heterosis, cuatro están formados con híbridos de la Narro y uno con un híbrido de Dekalb como macho, y como hembra dos híbridos de Dekalb.

En el Cuadro 4 se presentan las estimaciones de ACG de los 13 híbridos comerciales, donde se observa que los mejores efectos positivos para rendimiento fueron obtenidos por los híbridos PP9539 con 1,168 t/ha, AN447 con 0,684 t/ha y AS910 con 0,530 t/ha. Las estimaciones de ACG para altura de planta, humedad de grano y días a floración revelaron que el híbrido PP9539 contribuye al la reducción de altura con -3,783 $\mathrm{cm}$, humedad de grano con $-1,436$ por ciento y días a floración con -2,643 días; el AN447 mantiene su altura con $0,217 \mathrm{~cm}$, reduce la humedad de grano con -1.218 por ciento y mantiene su días a floración con 0,038 días, y por ultimo, el AS910 el cual contribuye al incremento de altura de planta en sus cruzas con $2,625 \mathrm{~cm}$, incrementa en un mínimo la humedad de grano con 0,118 por ciento y decrementa los días a floración -0,598 días.

Además de los análisis de varianza dialélicos también se realizo un análisis de varianza bajo el modelo II de Gardner-Eberhart (1966) para rendimiento (Cuadro 5) donde se observa que hubo diferencias significativas para todas la fuentes de variación, excepto para la heterosis promedio por ambiente. Al examinar las fuentes de variación mas importantes de este análisis, la significancia de heterosis por ambiente indica que la hetero-

Cuadro 4. Aptitud combinatoria general (ACG) entre 13 híbridos comerciales de maíz en tres ambientes, dos en Celaya Gto. y uno en Sandia N.L. en el año de 1996. Coahuila, México.

\begin{tabular}{lccr}
\hline Híbrido & $\begin{array}{c}\text { Rendimiento } \\
\text { t/ ha }\end{array}$ & $\begin{array}{c}\text { Altura de la planta } \\
\text { cm }\end{array}$ & $\begin{array}{c}\text { Humedad de grano } \\
\text { \% }\end{array}$ \\
\hline C220 & $-0,616$ & $-3,510$ & 0,581 \\
C221 & $-0,962$ & $-2,783$ & 1,236 \\
A7500 & $-0,702$ & $-3,601$ & 0,532 \\
PP9603 & $-0,677$ & $-6,010$ & $-0,086$ \\
PP9539 & 1,168 & $-3,738$ & $-1,436$ \\
PP9538 & 0,142 & 1,717 & $-0,905$ \\
AN447 & 0,684 & 0,217 & $-1,218$ \\
AN454 & $-0,081$ & 6,853 & 0,441 \\
AN450 & 0,044 & 0,626 & 1,001 \\
AN453 & $-0,087$ & 5,762 & $-2,916$ \\
AS910 & 0,530 & 2,625 & $-1,643$ \\
AS4450 & 0,104 & 1,671 & 0,038 \\
AS948 & 0,452 & 0,17 & 2,129 \\
\end{tabular}


Cuadro 5. Cuadrados medios del análisis de varianza combinado de 13 híbridos de maíz y sus 78 cruzas posibles para la características rendimiento, analizados bajo el modelo II de Gardner y Eberhart (1966) en los tres ambientes de evaluación. Coahuila, México. 1996-97.

\begin{tabular}{lcc}
\hline \multicolumn{1}{c}{ F.V } & G.L. & Cuadrados medios \\
\hline Ambientes & 2 & $253.998^{* *}$ \\
Rep/Ambientes & 3 & 9.313 \\
Cruzas e híbridos & 90 & $14.141^{* *}$ \\
Híbridos & 12 & $38.780^{* *}$ \\
Heterosis & 78 & $10.346^{* *}$ \\
Het. promedio & 1 & $62.928^{* *}$ \\
Het. varietal & 12 & $24.450^{* *}$ \\
Het. específica. & 65 & $6.931^{* *}$ \\
Cruzas e Hib. x ambientes & 180 & $8.769^{* *}$ \\
Hib. x amb. & 24 & $34.729^{* *}$ \\
Het. x amb. & 156 & $4.775^{* *}$ \\
Het. prom. x amb. & 2 & 3.356 \\
Het. var. amb. & 24 & $5.151^{* *}$ \\
Het. esp. x amb. & 130 & $4.727^{* *}$ \\
Error & 270 & 2.748 \\
\hline
\end{tabular}

*,** significativo al 0,05 y 0,01 respectivamente.

sis no es estable a través de los ambientes, al desglosar la heterosis se observa que la heterosis especifica por ambiente contribuye más a la heterosis quedando en segundo termino la heterosis varietal por ambiente y por último la heterosis promedio por ambiente. En lo que respecta a la heterosis promedio por ambiente, además que también es una comparación de cruzas vs. padres donde los padres obtuvieron un rendimiento de 15.605 t/ha y las cruzas $14.235 \mathrm{t} /$ ha con una diferencia de 1.307 $\mathrm{t} /$ ha (heterosis promedio por ambientes) siendo no significativa, indica que esta se mantiene estable a través de los ambientes. La significancia de la heterosis varietal por ambiente indica que la heterosis de las variedades no fueron estables a través de los ambientes, además de que no fue muy alta debido probablemente a que varios de los híbridos usados están emparentados, sin embargo, hubo un híbrido con la más alta heterosis en los tres ambientes (Cuadro 6). En lo que respecta a la heterosis específica por ambiente, la cual fue altamente significativa, revela que las heterosis de las cruzas (o ACE de las mismas) es diferente a través de las localidades, sin embargo se detectaron cruzas las cuales su heterosis especifica (o ACE) se mantiene a través de los ambientes (Cuadro 1).

En el Cuadro 6 se presentan las mejores cruzas, los híbridos, su rendimiento, heterosis promedio, aptitud combinatoria general y efecto varietal. Se observa que el híbrido con mejor heterosis varietal fue el PP9539 $(2,269 \%)$ mismo que también presento la mejor aptitud combinatoria general (1.168 t/ha) relación que no se presento en los demás híbridos, indicando que la heterosis no es totalmente controlada por genes dominantes, sino que también es controlada por genes aditivos en cierto grado, pero esto no es común en la mayoría de los híbridos, el segundo y tercer lugar fueron obtenidos por los híbridos A7500 y C220 con 1,107\% y 1,070\% respectivamente.

\section{Análisis de relación genética}

En el Cuadro 7 se muestran las distancias genéticas basadas en los datos del rendimiento (Celaya I y Celaya II) de las cruzas entre los híbridos de acuerdo a la fórmula de Troyer (1988) en la parte superior de la diagonal, y de los datos de la heterosis en base al progenitor medio en la parte inferior. Se observa que los híbridos con las distancias más pequeñas son los que obtuvieron las heterosis más bajas, esto se puede observar en los híbridos AN450 y AS910, los cuales tuvieron la distancia genética más baja $(0.033)$ y también la heterosis mas baja $(-47,68 \%)$, otro de los híbridos que mostraron resultados similares fueron el AN453 y el AS948, los cuales tuvieron una distancia genética de $(0,218)$ y una heterosis de $(-35,34 \%)$, por el contrario. los híbridos con mayor distancia genética AN454 y A7500 con (1.418), obtuvieron también la heterosis mas grande $(12,26 \%)$, datos similares fueron obtenidos por los híbridos PP9539 y PP9603 que a pesar de pertenecer a la misma compañía tuvieron una distancia genética de (1.326) y una heterosis de $(9,83$ por ciento).

Con los datos obtenidos de las distancias genéticas se realizó un análisis de conglomerados o (Cluster Analysis) cuyos resultados se presentan en la Figura $1 \mathrm{El}$ análisis de conglomerados permite obtener una representación gráfica de los datos del Cuadro 6 en forma de dendograma. El agrupamiento de los híbridos confirma lo señalado por las distancias genéticas anteriormente, de que el AS910 y el AN450 son 
Cuadro 6. Rendimiento, heterosis y aptitud combinatoria específica de las mejores 13 cruzas y rendimiento, heterosis varietal, aptitud combinatoria general y efecto varietal de los híbridos de maíz evaluados en tres ambientes. Coahuila, México. 1996-97.

\begin{tabular}{llcccccrrr}
\hline Cruza & & $\begin{array}{c}\text { Rend. } \\
\text { t/ha }\end{array}$ & $\begin{array}{c}\text { Heterosis } \\
\text { t/ha }\end{array}$ & $\begin{array}{c}\text { A.C.E. } \\
\text { t/ha }\end{array}$ & Híbrido & $\begin{array}{c}\text { Rend. } \\
\text { t/ha }\end{array}$ & $\begin{array}{r}\text { Heterosis } \\
\text { t/ha }\end{array}$ & $\begin{array}{c}\text { A.C.G. } \\
\text { t/ha }\end{array}$ & $\begin{array}{c}\text { EF. VAR. } \\
\text { t/ha }\end{array}$ \\
\hline \multirow{2}{*}{ AS910 } & AS4450 & 17.538 & 0.163 & 2.669 & C220 & 12.233 & 1.070 & $-0,616$ & $-3,372$ \\
PP9538 & AS948 & 14.463 & 1.178 & 2.633 & C221 & 14.212 & $-0,266$ & $-0,962$ & $-1,393$ \\
PP9539 & AN453 & 17.355 & 1.970 & 2.038 & A7500 & 11.987 & 1.107 & $-0,702$ & $-3,618$ \\
PP9539 & AN450 & 17.188 & 1.274 & 1.741 & PP9603 & 14.949 & 0,349 & $-0,677$ & $-0,656$ \\
PP9539 & AN447 & 17.072 & 1.702 & 0,985 & PP9539 & 13.402 & 2.269 & 1,168 & $-2,203$ \\
AN454 & AS948 & 16.052 & $-0,543$ & 1.445 & PP9538 & 15.016 & 0,436 & 0,142 & $-0,589$ \\
AN447 & AS948 & 16.033 & $-1,413$ & 0,661 & AN447 & 17.339 & $-0,183$ & 0,684 & 1,734 \\
PP9539 & AS948 & 16.000 & 0,523 & 0,144 & AN454 & 15.636 & $-0,097$ & $-0,081$ & 0,031 \\
PP9539 & AN454 & 15.996 & 1.476 & 0,673 & AN450 & 18.426 & $-1,366$ & 0,044 & 2,821 \\
PP9603 & PP9539 & 15.952 & 1.776 & 1.225 & AN453 & 17.367 & $-0,967$ & $-0,087$ & 1,762 \\
AN450 & AS4450 & 15.929 & $-0,906$ & 1.546 & AS910 & 19.505 & $-1,421$ & 0,530 & 3.900 \\
PP9539 & AS910 & 15.624 & $-0,830$ & $-0,310$ & AS4450 & 15.245 & 0,284 & 0,104 & $-0,360$ \\
C220 & AN447 & 15.618 & 0,832 & 1.315 & AS948 & 17.553 & $-0,521$ & 0,452 & 1,948 \\
\hline
\end{tabular}

Media de padres $15.605 \mathrm{t} / \mathrm{ha}$

Heterosis promedio $-1,370 \mathrm{t} / \mathrm{ha}$

Media de cruzas $14.235 \mathrm{t} / \mathrm{ha}$

CUADRO 7. Estimaciones de diversidad genética entre 13 híbridos comerciales evaluados en las localidades (Celaya I y Celaya II Gto), en el año de 1996 (arriba de la diagonal), y heterosis (abajo de la diagonal). Coahuila, México. 1996-97

\begin{tabular}{|c|c|c|c|c|c|c|c|c|c|c|c|c|c|}
\hline Padres & $\mathrm{C} 220$ & $\mathrm{C} 221$ & $\mathbf{A 7 5 0 0}$ & PP9603 & PP9539 & PP9538 & AN447 & AN454 & AN450 & AN453 & AS910 & AS4450 & AS948 \\
\hline $\mathrm{C} 220$ & & 0,636 & 1,022 & 0,831 & 1,096 & 0,909 & 1,112 & 1,001 & 0,849 & 0,934 & 0,982 & 0,821 & 0,739 \\
\hline $\mathrm{C} 221$ & $-13,85$ & & 1,212 & 0,928 & 1,119 & 0,671 & 0,868 & 1,242 & 0,803 & 0,947 & 0,927 & 0,716 & 0,809 \\
\hline A7500 & 0,73 & 6,663 & & 0,618 & 1,152 & 1,025 & 1,031 & 1,418 & 0,855 & 1,076 & 0,981 & 1,007 & 1,170 \\
\hline PP9603 & $-6,00$ & $-2,46$ & $-14,62$ & & 1,326 & 0,854 & 0,730 & 0,806 & 0,700 & 0,539 & 0,792 & 0,790 & 0,660 \\
\hline РP9539 & 3,11 & 3,83 & $-4,85$ & 9,83 & & 1,088 & 1,269 & 1,354 & 1,082 & 1,140 & 0,949 & 0,721 & 1,086 \\
\hline PP9538 & $-3,15$ & $-12,36$ & 0,82 & $-5,14$ & 2,86 & & 0,929 & 0,990 & 0,886 & 0,888 & 0,662 & 0,522 & 1,160 \\
\hline AN447 & 3,61 & $-4,63$ & 1,01 & $-9,90$ & 8,25 & $-2,44$ & & 0,500 & 0,421 & 0,510 & 0,397 & 0,809 & 0,478 \\
\hline AN454 & 0,03 & 7,48 & 12,26 & $-6,92$ & 10,56 & $-0,33$ & $-20,03$ & & 0,462 & 0,523 & 0,552 & 0,974 & 0,859 \\
\hline AN450 & $-5,30$ & $-7,03$ & $-5,08$ & $-11,14$ & 2,67 & $-3,97$ & $-23,97$ & $-21,92$ & & 0,267 & 0,033 & 0,841 & 0,306 \\
\hline AN453 & $-2,24$ & $-1,79$ & 2,48 & $-18,92$ & 4,48 & $-3,88$ & $-19,56$ & $-18,93$ & $-32,44$ & & 0,565 & 0,371 & 0,218 \\
\hline AS910 & $-0,62$ & $-2,50$ & $-0,64$ & $-7,84$ & $-1,72$ & $-12,71$ & $-25,23$ & $-17,61$ & $-47,68$ & $-17,01$ & & 1,020 & 0,394 \\
\hline AS4450 & $-6,38$ & $-10,49$ & 0,23 & $-7,56$ & $-10,27$ & $-18,98$ & $-6,81$ & $-0,87$ & $-5,59$ & $-26,62$ & 0,67 & & 0,282 \\
\hline AS948 & $-9,57$ & $-6,81$ & 5,37 & $-12,80$ & 2,84 & 5,07 & $-21,12$ & $-4,94$ & $-30,15$ & $-35,34$ & $-25,38$ & $-31,55$ & \\
\hline
\end{tabular}

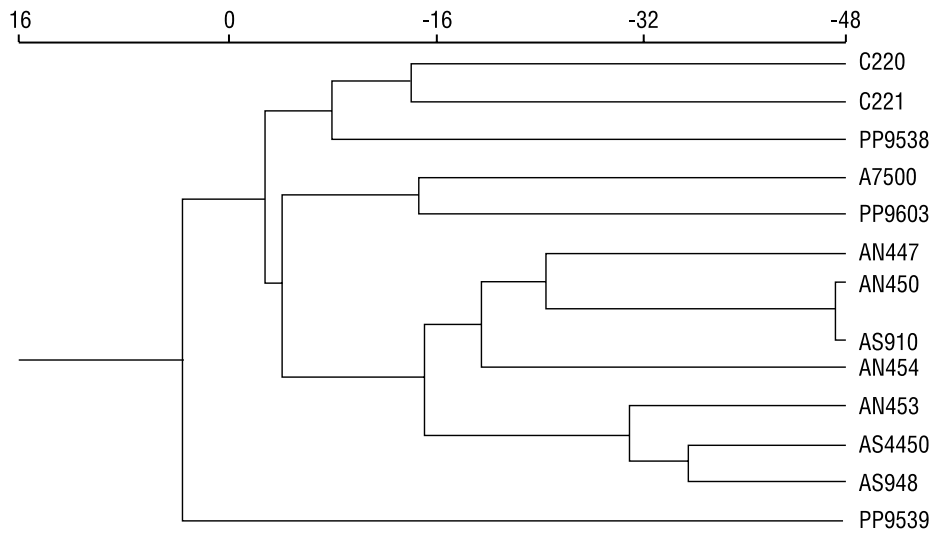

Figura 1. Agrupamiento de 13 híbridos comerciales de maíz en base a heterosis del rendimiento. Coahuila, México. 1996-97 
los híbridos mas relacionados genéticamente y de forma similar el AN453 y AS948. También se puede observar como el AN447, AN450, AS910, AN454, AN453, AS948 y AS4450 forman un mismo grupo indicando que estos híbridos están relacionados unos en mayor y otros en menor magnitud genéticamente; también se pude ver como los híbridos C220 y C221 forman un grupo bien definido. a una distancia de 0,636 lo cual es lógico ya que estos dos híbridos pertenecen a una misma compañía y pudiera ser que tienen uno o dos progenitores en común, otro grupo lo forman el A7500 y PP9603, los cuales a pesar de ser de diferente compañía es posible que compartan alguna similitud genética, y por último el híbrido PP9539 el cual se observa que no esta relacionado con ninguno de los híbridos incluidos en este estudio; lo que concuerda con los resultados de ACG para rendimiento, ya que fue el híbrido con mayor ACG.

Con los datos de la heterosis, también se realizó un análisis de conglomerados (Figura 2) donde se obtuvo un dendograma similar al obtenido con las distancias genéticas, además se realizó un análisis de correlación entre las distancia genéticas y la heterosis (Figura 3) donde se obtuvo una correlación de $r=0,98$, concluyendo que tanto la estimación de heterosis como la distancia genética son importantes para determinar patrones heteróticos entre materiales (híbridos, líneas y poblaciones) para el uso de estos en programas de mejoramiento y que la heterosis es un parámetro importante en un programa de mejoramiento.

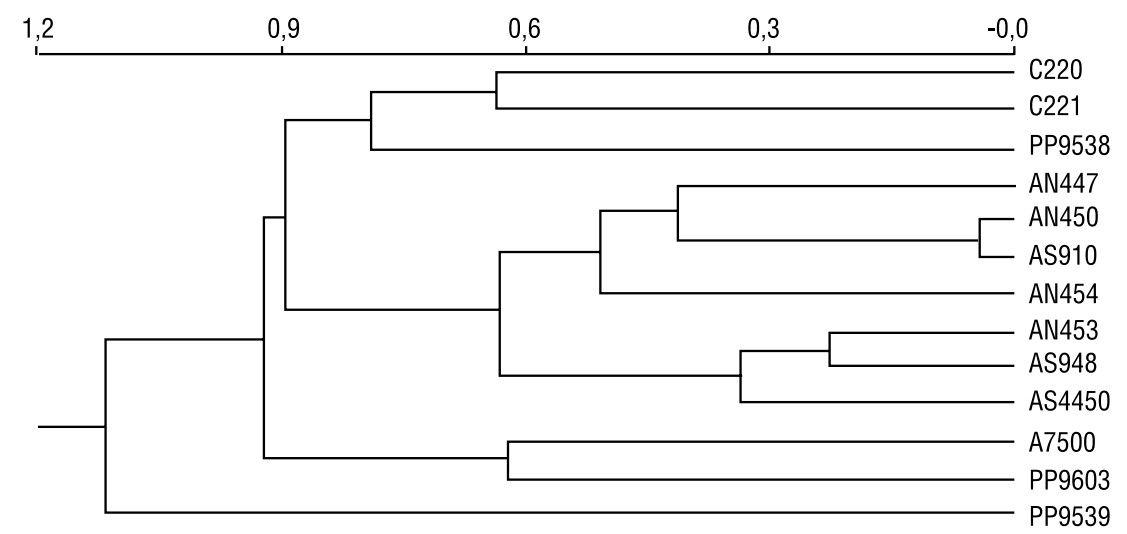

Figura 2. Agrupamiento de 13 híbridos comerciales de maíz en base a distancias genéticas del rendimiento. Coahuila, México. 1996-97.

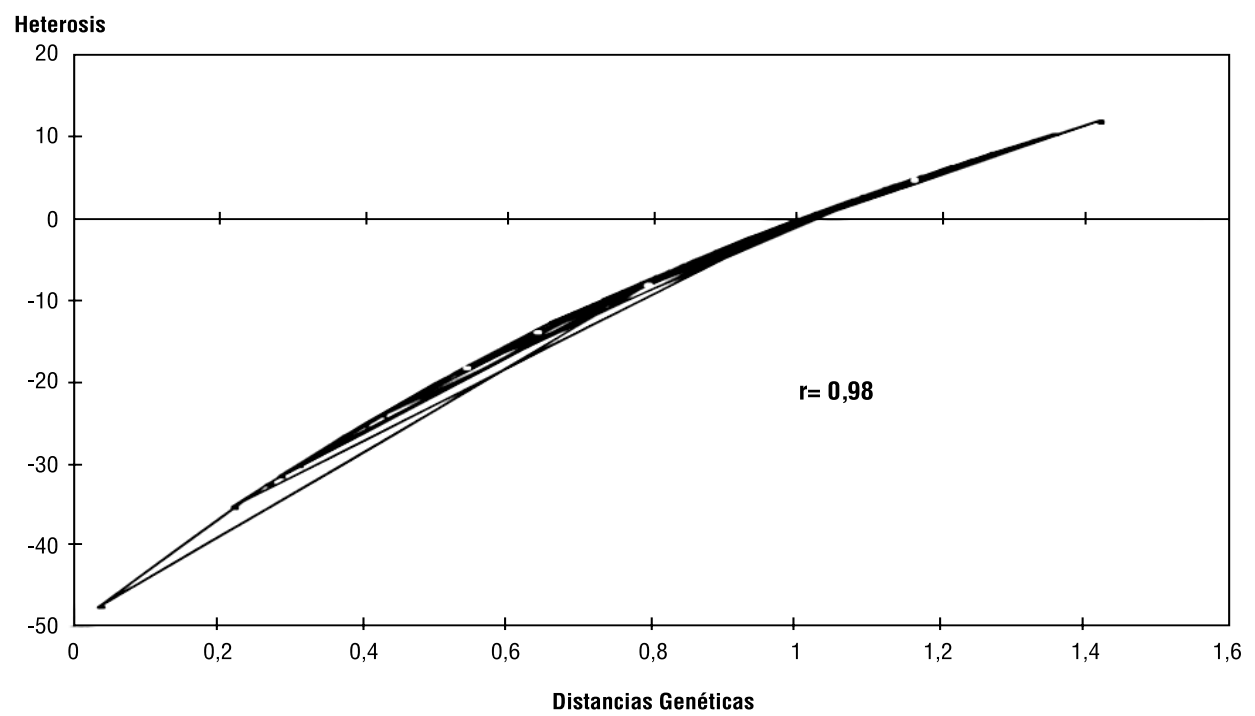

Figura 3. Correlación entre distancias genéticas y heterosis de los 13 híbridos comerciales de maíz. Coahuila, México. 1996-97. 


\section{CONCLUSIONES}

De las mejores cruzas en $\mathrm{F}_{2}$ se pueden derivar líneas prometedoras para la formación de híbridos y sintéticos.

Los híbridos que forman las cruzas con mayor heterosis se pueden usar en un programa de mejoramiento de líneas formadoras de cruzas simples aprovechando el patrón heterótico de estos (selección gamética y pedigrí)

Los híbridos con mayor ACG y heterosis se pueden usar para el mejoramiento de líneas.

\section{LITERATURA CITADA}

CASTRO, G. M.; GARDNER, C. O.; LONNQUIST, J. H. 1968. Cumulative gene efects and the nature of heterosis in maize crosses involving genetically diverse races. Crop. Sci. 8:91-101

CORTEZ, M. H.; RODRIGUEZ C., A.; GUTIÉRREZ G.; DURON Y, J.; GIRON C., R.; Oyervides G., M. 1985. Evaluation of broad-base improved population of maize (Zea mays L.) Y Cumulative gene efects and heterosis. U.A.A.A.N. Buenavista Saltillo Coah.

EVERIT, B. 1980 Cluster analysis. Second edition. Halsted press. New York.

GARDNER, C.O.; EBERHART S.A. 1966. Analysis and interpretation of the variety cross diallel end related populations. Biometrics 22:439-452

GARZA, A.; FLEMING, A. A.; BROWNE, E.B.1962. A method of identifying maize hjybrids with the same pedi- gree and the effect of varying number of lines in common on contrast among hybrid and sib populations . Crop Sci. 2:371-374

GONZALEZ, L. J. S. 1996. Integración de un patrón heterótico a partir de un dialélico de diez líneas de maíz subtropicales. Tesis M.C. U.A.A.A.N. Buenavista Saltillo, Coahuila México.

GRIFFING, B. 1956. Concept of general and specific combining ability in relation to diallel crossing system. Australian J. Biol. Sci. 9, 463-93.

LEE, M.; GODSHALK, E.B.; LAMKEY, K.R.; WOODMAN, W.W. 1989. Associacion of restriction fragment length polymorphisms among maize inbreds with agronomic performance of their crosses. Crop. Sci. 29:1067-1071.

MUMM R. H.; DUDLEY, J. W. 1994. A classification of 148 U. S. Maize inbreds: I. Cluster analysis based on RFLPs Crop. Sci. 34:842-851

SMITH J.S.C.; SMITH, O.S. 1989. Comparison of heterosis among Hybrids as a measure of hybrid relatedness with that to be expected on the bases of pedigree. Maize Genet. Coop. Newl. 63:86-87

SMITH, O. S. ; SMITH, J.S.C.; BOWEN, S.L. TENBORG, R. A.; WALL, S.J. . 1990. Similarities among a group of elite maize inbreds as measured by pedigree, $\mathrm{F}_{1}$, grain yield, grain yield heterosis, and RFLPs. Theor. Appl. Genet. 80:833-840.

TROYER, A.F. ; OPENSHAW, S.J.; KNITTLE, K:H . 1988. Mesaurement of genetic diversity among popular corn hybrids. Crop Sci. 28: 481-485. 